\title{
Ethnicity influences disease characteristics and symptom severity in allergic rhinitis patients in Malaysia
}

\begin{abstract}
Background: The number of available reports regarding the influence of ethnicity on clinical features of allergic rhinitis (AR), especially disease severity in tropical climates, is limited. We aimed to compare clinical parameters and disease severity in AR patients of different ethnicities.

Methods: Malay, Chinese, and Indian AR patients $(\mathrm{n}=138)$ with confirmed sensitivity to Dermatophagoides pteronyssinus, Dematophagoides farinae, and Blomia tropicalis were tested for mite-specific immunoglobulin E (sIgE) levels. A detailed questionnaire was used to collect data on nasal symptom score (NSS), ocular symptom score (OSS), sum of symptoms score (SSS), quality of life score (QLS), symptomatic control score (SCS), and total sum of scores (TSS) and correlate the derived data with patients' demography, mite-polysensitivity, and sIgE levels.

Results: AR-related symptoms were most severe in Malays and least in Chinese ( $\mathrm{p}<0.01$ ). Age $(r=0.516$ to $0.673, p<0.05)$ and duration of AR $(r=0.635$ to $0.726, p<0.01)$ correlated positively with severity domains (NSS, SSS, QLS, and TSS) in Chinese. Duration of concurrent allergies was highest in Malays ( $\mathrm{p}<0.05)$. Polysensitivity predicted increased sIgE levels in Malays ( $r=0.464$ to $0.551, p<0.01)$ and Indians ( $r=0.541$ to $0.645, p<0.05)$ but affected NSS, SSS, and TSS only in Indians $(r=0.216$ to $0.376, p<0.05)$. sIgE levels were lowest among Chinese but correlated strongly with NSS, OSS, SSS, and TSS ( $\mathrm{r}=0408$ to $0.898, \mathrm{p}<$ $0.05)$.

Conclusion: Clinical parameters in AR may be influenced by race. Symptoms were most severe among Malays but did not correlate with other variables examined. Although Indian ethnicity did not impact disease severity, duration of concurrent allergies and mite-polysensitivity was associated with more severe disease. Age, duration of disease, and sIgE levels may be useful indicators of disease severity in Chinese.
\end{abstract}

Keyword: Allergic rhinitis; Mite; Polysensitivity; Race; Serum IgE; Severity 